\title{
Immunotherapy-Induced Acute Hepatitis in the Elderly: The Case of a Patient with Urothelial Carcinoma and a Review of the Literature
}

Research Article

Ioannou Alexandros ${ }^{1 *}$, Papaxoinis Georgios², Dimitroulopoulos Dimitrios ${ }^{1}$,

Tryfonopoulos Dimitrios², Gouveris Panagiotis²

${ }^{1}$ Gastroenterology Department, Agios Savvas Cancer Hospital, 11522 Athens,

Greece

22nd Department of Medical Oncology, Agios Savvas Cancer Hospital

Received 11 April 2021; Accepted 15 July 2021

\begin{abstract}
Cancer immunotherapy is an emerging beneficial treatment for cancer that acts by activating the immune system to produce antitumour effects. In particular, immune checkpoint therapy has recently provided novel strategies for cancer treatments. Nevertheless, these new therapeutic approaches have introduced immune-related adverse events to clinical practice. In the elderly, checkpoint inhibitors might have limited efficacy because of immunosenescence. Limited literature data demonstrate a higher incidence of irAEs and an earlier discontinuation of immunotherapy in these patients. We report the case of an 89-year-old male patient with metastatic urothelial carcinoma, who presented with Grade 4 immune-related hepatitis after receiving pembrolizumab treatment.
\end{abstract}

Keywords: Immunotherapy-related adverse events • pembrolizumab • immunotherapy-related hepatitis • elderly

\section{Introduction}

Monoclonal antibodies targeting cytotoxic T lymphocyteassociated antigen 4 (CTLA4) and the programmed death-1 receptor (PD-1) or its ligand PD-L1 constitute a standard of care for an increasing number of indications. Pembrolizumab is an antiprogrammed cell death-1 (PD-1) checkpoint inhibitor. It was first approved for the treatment of advanced melanoma and metastatic nonsmall-cell lung carcinoma (NSCLC) and subsequently was approved for several other malignancies like relapsed or refractory classical Hodgkin's lymphoma, locally advanced or metastatic urothelial carcinoma, recurrent or metastatic head and neck squamous cell carcinoma, and mismatch-repair-deficient solid tumours. PD-1 blockade constitutes a cornerstone in modern cancer immunotherapy ${ }^{[1]}$.

The introduction of immune checkpoint inhibitors in everyday clinical practice requires the oncologist to collaborate with doctors from other specialities to deal with so-called immune-related adverse events. Multiple organs of the body can be affected. Regarding the liver, immune-related toxicities could range from asymptomatic transaminase elevations to rare, more severe and even life-threatening forms of acute hepatitis ${ }^{[1-3]}$.

With the advent of immune checkpoint inhibitors, a new type of drug-induced liver injury (DILI) has emerged. Hepatitis induced by immune checkpoint inhibitors is usually asymptomatic and detected with routine blood monitoring that could reveal isolated elevations of liver transaminases. Liver biopsy should be considered in more severe cases. The histomorphologic findings of panlobular hepatitis with a predominantly CD8positive inflammatory infiltrate are, in most patients, indistinguishable from typical features seen in classical autoimmune hepatitis. Cholestasis consistent with a portal mononuclear infiltrate can be observed in patients with severe hepatic immune-mediate Adverse Events (imAEs) following treatment with ipilimumab ${ }^{[3-8]}$. 
In the elderly population, there is no evidence regarding the efficacy and toxicity of immune checkpoint inhibitors. Older patients included in therapeutic trials are usually not representative of the general population because of selective criteria of inclusion and more frequent discontinuation of treatment than younger patients $^{[9]}$.

We report the case of an 89-year-old male patient with metastatic urothelial carcinoma, who presented with Grade 4 immune-related hepatitis after receiving pembrolizumab treatment.

\section{Case report}

An 89-year-old male patient with a history of urothelial bladder cancer presented to our emergency room (ER) unit with macroscopic haematuria, fatigue, and anorexia. Blood exams revealed Grade 4 hepatitis, with ALT 982 U/I, AST $957 \mathrm{U} / \mathrm{l}$, alkaline phosphatase $173 \mathrm{U} / \mathrm{l}$, Y-GT $189 \mathrm{U} / \mathrm{l}$, and bilirubin 0,38U/l. Full blood count values were within normal ranges. There was no PT or APTT prolongation. The patient was evaluated by an urologist, who attributed haematuria to the known malignancy and recommended observation and intravenous fluid therapy. The patient was admitted to our Department of Medical Oncology for further investigation and support.

The patient had been diagnosed with muscleinvasive urothelial carcinoma five years earlier. He was a non cystectomy candidate. Therefore, he had been treated with concurrent chemoradiotherapy. Four months earlier, CT follow-up revealed bone and pelvic lymph node metastases. The patient was started on first-line therapy with pembrolizumab 200 mg every three weeks. Before the initiation of pembrolizumab treatment, liver function blood tests were normal. Liver tests were also normal before the second and third cycle of pembrolizumab (every three weeks). He received the third treatment cycle a week before his appearance in our ER unit.

During the patient's hospitalisation in our department, an abdominal CT was performed, and it was negative for liver metastases or any other hepatic parenchyma lesions (Fig. 1). A pelvis CT revealed stable disease regarding pelvic lymph node metastases. Blood tests of viral hepatitis were negative. The patient denied alcohol consumption.

According to ASCO guidelines for the management of liver imAEs, the patient was treated with $2 \mathrm{mg} / \mathrm{kg} /$ day prednisone. There was a gradual decrease in ALT and AST levels (Fig. 2). Haematuria resolved in the first days of hospitalisation. The patient was dismissed from our department a week after his admission with normal
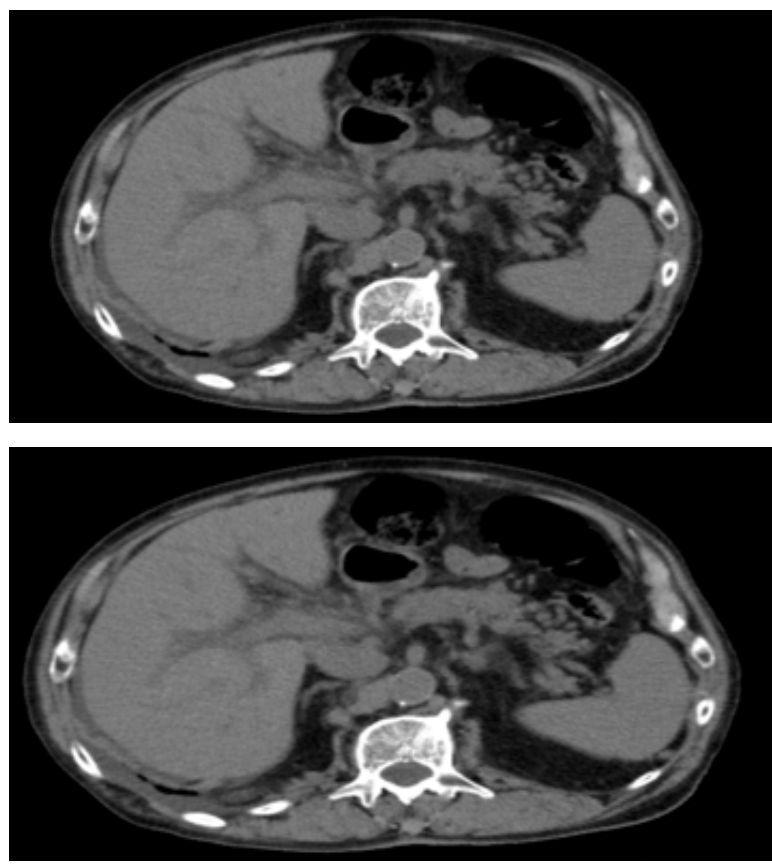

Figure 1: Abdominal CT performed during hospitalization.

levels of ALT and AST and with recommendations for permanent discontinuation of pembrolizumab and for corticosteroid tapering over a period of three more weeks.

\section{Discussion}

Pembrolizumab is a humanised monoclonal antibody. It binds to the programmed cell death-1 (PD-1) receptor, which is expressed on the surface of cytotoxic T-cells and blocks its interaction with ligands PD-L1 and PDL2, which are expressed in antigen-presenting cells and may be expressed by tumours or other cells in the tumour microenvironment. The PD-1 receptor is a negative regulator of $\mathrm{T}$-cell activity and is involved in the control of T-cell immune responses. It protects tumour cells from immune system attack. Pembrolizumab potentiates T-cell responses, including antitumour responses, through blockade of PD-1 binding to PD-L1 and PD-L2. By blocking the PD-L1 and PD-L2 pathway, pembrolizumab helps T-cells remain activated and ready to attack tumour cells ${ }^{[10]}$.

PD-1 inhibitors could cause severe immune-related hepatitis, the mechanism of which still has not been fully determined. In normal physiological conditions, the liver immune condition remains in tolerance. Sinusoidal endothelial cells of the liver express high levels of PD-L1 to avert overimmune response and protect normal liver 


\section{ALT, AST AND BLOOD BILIRUBIN LEVELS DURING HOSPITALIZATION}

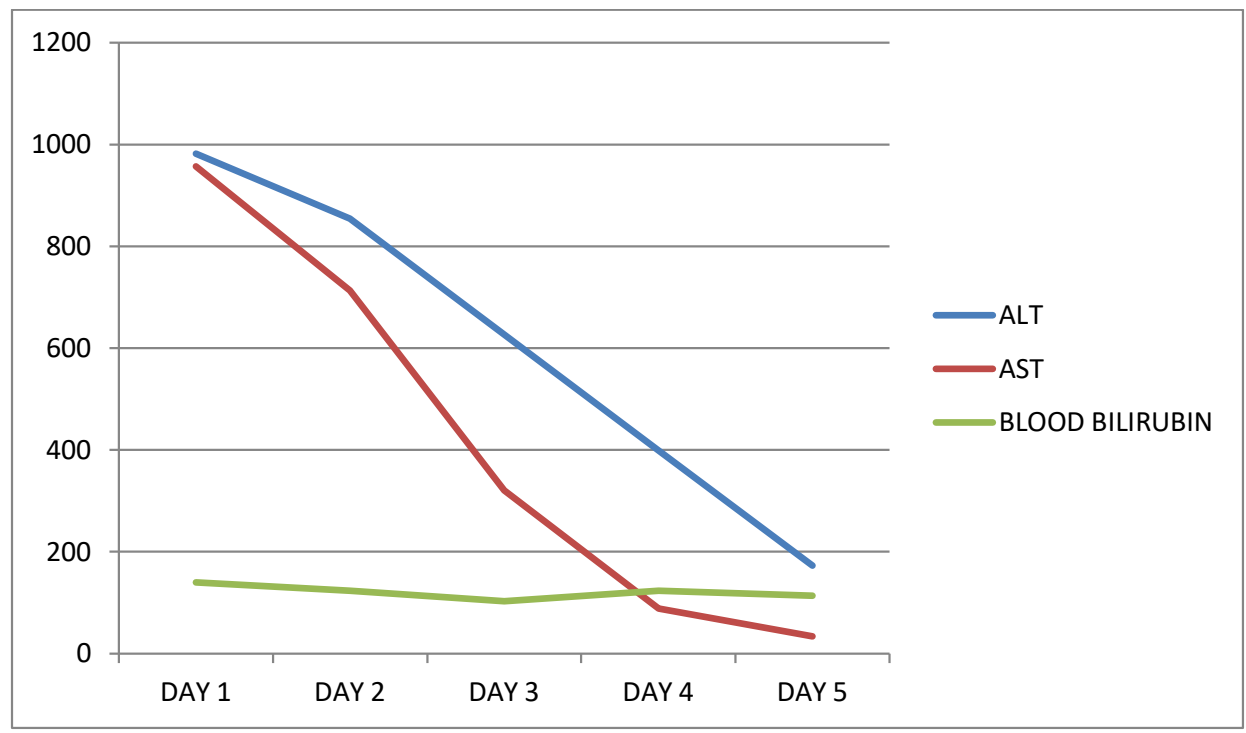

Figure 2: ALT, AST, and blood bilirubin levels during hospitalisation.

tissue from immune damage through the activation of the PD-1/PD-L1 signalling pathway ${ }^{[11,12]}$. Pembrolizumab blocks the PD-1/PD-L1 signalling pathway and reverses the depletion of T-cell function leading to an imbalance in the tolerance of the hepatic immune system by decreasing inhibitory regulation of the autoimmune response, and triggers an unchecked immune response, which causes significant liver dysfunction ${ }^{[11,12]}$.

In the general population, hepatotoxicity has been reported to occur in $2 \%$ to $10 \%$ of patients treated with ipilimumab, nivolumab, and pembrolizumab monotherapy. Onset develops predominantly within the first 5 to 12 weeks after treatment initiation, but the median time of onset is at about 6 weeks after treatment initiation $^{[7,15,16]}$. The pooled incidence of immune-related hepatotoxicity is estimated at $3 \%$ to $9 \%$ for ipilimumab and between $0.7 \%$ and $1.8 \%$ for PD-1/PD-L1 inhibitors ${ }^{[17,18]}$. According to other references, hepatic irAEs seem to have an almost equal incidence in patients receiving ipilimumab and in those receiving anti-PD-1 antibodies as monotherapies (occurring in $5-10 \%$ of patients) albeit with a tendency towards greater severity in patients receiving ipilimumab ${ }^{[1]}$. If we focus on pembrolizumab studies, according to European Medicines Agency (EMA), immune-related hepatitis occurred in 39 (0.8\%) patients, including Grade 2, 3, or 4 cases in $7(0.1 \%)$, $26(0.5 \%)$ and $4(<0.1 \%)$ patients, respectively, receiving pembrolizumab. The median time to onset of hepatitis was 2.8 months (range $=8$ days -21.4 months). The median duration was 1.1 months (range = 1 day-20.9 months). Hepatitis led to discontinuation of pembrolizumab in $14(0.3 \%)$ patients. Hepatitis resolved in 27 patients. Additionally, EMA reveals that in patients treated with pembrolizumab monotherapy, the proportion of patients who experienced a shift from baseline to a Grade 3 or 4 was $2.6 \%$ for AST increased and $2.3 \%$ for ALT increased ${ }^{[10]}$.

In older patients, it is possible that checkpoint inhibitors have limited efficacy because of immunosenescence. An ageing immune system consists of a declining number of CD4+ naive T-cells and an increased pool of differentiated CD8+ T-cells ${ }^{[13]}$. Additionally, the number of circulating and intratumoural myeloid derived suppressor cells increases. Consequently, T-cell function could decline and cause defective responsiveness to immune-related therapies ${ }^{[14]}$. In the elderly, randomised trials rarely studied these outcomes because of the reduced functional reserves and comorbidities related to age. A limited subgroup analysis of trial data demonstrates a higher incidence of imAEs and an earlier discontinuation of immunotherapy in the elderly ${ }^{[19,20]}$. Gomez et al., in the ELDERS study preliminary results, compared elderly to non-elderly patients regarding the incidence of imAEs. Analysis of comorbidity score, geriatric assessment and incidence of imAEs concluded that there is no 
Table 1: Common terminology criteria for adverse hepatic immunemediate adverse events [24].

\begin{tabular}{l|cccc}
\hline & GRADE 1 & GRADE 2 & GRADE 3 & GRADE 4 \\
\hline \multirow{2}{*}{ ALT } & $>U L N-3.0$ & $>3.0-5.0 \times$ & $>5.0-20.0$ & $>20.0$ \\
& $x \cup L N$ & $U L N$ & $x \cup L N$ & $x \cup L N$ \\
\multirow{2}{*}{ AST } & $>U L N-3.0$ & $>3.0-5.0 x$ & $>5.0-20.0$ & $>20.0$ \\
& $x U L N$ & $U L N$ & $x U L N$ & $x \cup L N$ \\
\hline Blood & $>U L N-1.5$ & $>1.5-3.0 x$ & $>3.0-10.0$ & $>10.0$ \\
Bilirubin & $x U L N$ & $U L N$ & $x U L N$ & $x \cup L N$ \\
\hline
\end{tabular}

Table 2: ASCO guidelines for the management of liver imAEs [12].

Management of Hepatitis imAE

Consider mycophenolate mofetil for steroid refractory im

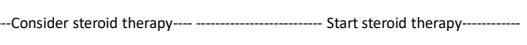



Monitorin

GRADE 1

\begin{tabular}{|c|}
\hline AMBULATORY HOSPITALIZED \\
\hline
\end{tabular}

statistically significant correlation. Regrettably, these results involved a small group of 32 patients included in this study and a limited follow-up of three months ${ }^{[21]}$.

Radiographic findings are not typically seen in patients with abnormal liver function tests. However, CT scans may show mild hepatomegaly, periportal oedema, or periportal lymphadenopathy. Biopsies of some patients with hepatotoxicity have revealed severe panlobular hepatitis with prominent perivenular infiltrate with endothelialitis, suggestive of predominant injury to hepatocytes. A primary biliary pattern with mild portal mononuclear infiltrate around proliferated bile ductules, suggestive of predominant injury to bile ducts, has also been reported in patients receiving ipilimumab ${ }^{[2,5,18]}$.

Pembrolizumab extends overall survival in metastatic urothelial carcinoma patients who underwent disease progression during or after platinum-based chemotherapy, as revealed in phase 3 of the KEYNOTE-045 study. In patients treated with pembrolizumab, $5.3 \%$ experienced any grade of AST and ALT elevation, and the proportions for Grade 3 or 4 AST and ALT elevation were $2.3 \%$ and $1.1 \%$, respectively ${ }^{[22]}$. Unfortunately, no specific subgroup analysis in older patients affected by urothelial carcinoma is available ${ }^{[19]}$. Pembrolizumab has also gained approval for the first-line treatment of patients with advanced urothelial carcinoma ineligible for cisplatin-containing chemotherapy. This was based on the results of KEYNOTE-052, a multicentre, singlearm, phase 2 study ${ }^{[23]}$. The results of phase 3 of the KEYNOTE-361 study of first-line pembrolizumab, with or without chemotherapy versus chemotherapy alone, are pending.

Common Terminology Criteria for Adverse Events classification includes four grades of hepatic immunemediated adverse events (imAEs; Table 1) ${ }^{[24]}$. If there is no apparent alternative aetiology, the next step, according to the ASCO guidelines for the management of imAEs, is a suitable corticosteroid treatment following an established algorithm. Hepatitis might persist and require prolonged or repeated corticosteroid tapers (four weeks treatment is a minimum period recommended), additional immunosuppression due to current management recommendations (Table 2), or both $^{[12]}$. In Grade 2, hepatic toxicity treatment with the checkpoint inhibitor should be withheld, and in Grade 3 or greater, hepatic toxicity should be permanently discontinued. Rarely, when there is no response to corticosteroid therapy, mycophenolate mofetil may be an additional medication to administer concurrently with corticosteroids. The use of antithymocyte globulin therapy has also been reported ${ }^{[2,3,25]}$. Unfortunately, literature data on the impact of immunotherapy-induced hepatitis on the outcome of cancer diseases are lacking.

The 89-year-old patient of our presentation experienced severe Grade 4 hepatitis one week after the third dose of pembrolizumab therapy, thus at about seven weeks from the start of treatment, which is close to the median time of onset reported in the literature. A diagnosis of pembrolizumab-induced immune-related hepatitis was made by excluding alternate aetiologies like viral or alcoholic hepatitis and other drug-induced liver injuries. Treatment with corticosteroids was successful and led to fast response and recovery. Because of the Grade 4 toxicity, the patient had to permanently discontinue immunotherapy.

\section{Authors Contribution}

All authors contributed to the study conception and design. Material preparation, data collection and analysis were performed by $A$. Ioannou and P. Gouveris. The first draft of the article was written by A. Ioannou, G.Papaxoinis and the other authors commented on previous versions of the article. Supervision was handled by D. Dimitroulopoulos and D. Tryfonopoulos. All authors read and approved the final article. 


\section{Conflict of interest}

The authors who have taken part in this study declare that they have no disclosure regarding funding or conflict of interest.

\section{References}

[1] Martins F, Sofiya L, Sykiotis GP, et al. Adverse effects of immune-checkpoint inhibitors: epidemiology, management and surveillance. Nat Rev Clin Oncol. 2019;May 15. doi:10.1038/s41571-019-0218-0.

[2] Postow M, Wolchok J. Patient selection criteria and toxicities associated with checkpoint inhibitor immunotherapy 2018. Uptodate.com.

[3] Haanen JBAG, Carbonnel F, Robert C, et al. Management of toxicities from immunotherapy: ESMO clinical practice guidelines for diagnosis, treatment and follow-up. Ann Oncol. 2017; Jul 1;28(suppl_4):iv119-iv142. doi:10.1093/annonc/ $\mathrm{mdx} 225$.

[4] Johncilla M, Misdraji J, Pratt DS, et al. Ipilimumab-associated hepatitis: clinicopathologic characterization in a series of 11 cases. Am J Surg Pathol. 2015;39.

[5] Kim KW, Ramaiya $\mathrm{NH}$, Krajewski KM, et al. Ipilimumab associated hepatitis: imaging and clinicopathological findings. Invest New Drugs. 2013;31.

[6] Karamchandani, D. M., Chetty, R. Immune checkpoint inhibitor-induced gastrointestinal and hepatic injury: pathologists' perspective. J Clin Pathol. 2018;71: 665-671.

[7] Ziemer M, Koukoulioti E, Beyer S, et al. Managing immune checkpoint-inhibitor-induced severe autoimmune-like hepatitis by liver-directed topical steroids. J Hepatology. 2017;66(3):657-659.

[8] Aivazian K., et al. Histopathology of pembrolizumabinduced hepatitis: a case report. Pathology. 2017;49(7):789-792.

[9] Friedman CF, Horvat TZ, Minehart J, et al. Efficacy and safety of checkpoint blockade for treatment of advanced melanoma (mel) in patients (pts) age 80 and older (80+). J Clin Oncology. 2016;34(15_ Suppl.):10009.

[10] European Medicine Agency Keytruda (pembrolizumab) authorization. https://www.ema. europa.eu/en/medicines/human/EPAR/keytruda.

[11] Wu Z, Lai L, Li M, Zhang L, Zhang W. Acute liver failure caused by pembrolizumab in a patient with pulmonary metastatic liver cancer: A case report. Medicine (Baltimore). 2017;96(51):e9431. doi:10.1097/MD.0000000000009431.

[12] Naidoo J, Page DB, Li BT, et al. Toxicities of the anti-PD-1 and anti-PD-L1 immune checkpoint antibodies. Ann Oncol. 2015;26:2375-2591.

[13] Elias R, Karantanos T, Sira E, et al. Immunotherapy comes of age: immune aging \& checkpoint inhibitors. J Geriatr Oncol. 2017;8(3):229-235.

[14] Daste A, Domblides C, Gross-Goupil M, et al. Immune checkpoint inhibitors and elderly people: a review. Eur J Cancer. 2017;82:155-166.

[15] Brahmer JR, Lacchetti C, Schneider BJ, et al. Management of immune related adverse events in patients treated with immune checkpoint inhibitor therapy: American Society of Clinical Oncology clinical practice guideline. J Clin Oncol. 2018;Jun 10;36(17):1714-1768. doi:10.1200/ JCO.2017.77.6385.

[16] Thompson JA, Schneider BJ, Brahmer J et al. Management of immunotherapy related toxicities, Version 2.2019-April 8, 2019. NCCN Clinical Practice Guidelines in Oncology.

[17] Suzman DL, Pelosof L, Rosenberg A, et al. Hepatotoxicity of immune checkpoint inhibitors: An evolving picture of risk associated with a vital class of immunotherapy agents. Liver Int, 2018;38:976-987.

[18] Kleiner DE, Berman D. Pathologic changes in ipilimumab-related hepatitis in patients with metastatic melanoma. Dig Dis Sci. 2012;57:2233.

[19] Holstein Y, Kapiteijn E, Bastiaannet E, van den Bos F, Portielje J, de Glas NA. Efficacy and adverse events of immunotherapy with checkpoint inhibitors in older patients with cancer. Drugs Aging. 2019 Oct;36(10):927-938. doi:10.1007/s40266-01900697-2. PMID: 31317421; PMCID: PMC6764930.

[20] Singh H, Kim G, Maher VE, et al. FDA subset analysis of the safety of nivolumab in elderly patients with advanced cancers. J Clin Oncology. 2016;34(15_Suppl.):10010.

[21] Gomes F, Woolley S, Califano R, et al. MA 1007 elderly lung cancer patients on immunotherapy: preliminary results from the ELDERS study. J Thorac Oncol. 2017;12(11):S1841-1842. 
[22] Bellmunt J, de Wit R, Vaughn DJ, et al. Pembrolizumab as second-line therapy for advanced urothelial carcinoma. N Engl J Med. 2017;376:1015.

[23] Balar AV, Castellano D, O'Donnell PH, et al. First-line pembrolizumab in cisplatin-ineligible patients with locally advanced and unresectable or metastatic urothelial cancer (KEYNOTE-052): a multicentre, single-arm, phase 2 study. Lancet Oncol 2017;18:1483.

[24] U.S. Department of Health and Human Services National Institutes of Health National Cancer Institute Common Terminology Criteria for Adverse Events (CTCAE). Version 5.0. November 27, 2017.

[25] Chmiel KD, Suan D, Liddle C. Resolution of severe ipilimumab-induced hepatitis after antithymocyte globulin therapy. J Clin Oncol. 2011;Mar;29(9):e237-40. 